\title{
THE STRUCTURE FUNCTION OF VARIABLE 1.4 GHz RADIO SOURCES BASED ON NVSS AND FIRST OBSERVATIONS
}

\author{
Eran O. OFEK ${ }^{1,3}$ and Dale A. Frail ${ }^{2}$ \\ ${ }^{1}$ Division of Physics, Mathematics and Astronomy, California Institute of Technology, Pasadena, CA 91125, USA \\ 2 National Radio Astronomy Observatory, Socorro, NM 87801, USA \\ Received 2011 March 6; accepted 2011 May 10; published 2011 July 27
}

\begin{abstract}
We augment the two widest/deepest $1.4 \mathrm{GHz}$ radio surveys, the NRAO VLA Sky Survey (NVSS) and the Faint Images of the Radio Sky at Twenty centimeters (FIRST), with the mean epoch in which each source was observed. We use these catalogs to search for unresolved sources that vary between the FIRST and NVSS epochs. We find 43 variable sources $(0.1 \%$ of the sources) that vary by more than $4 \sigma$, and we construct the mean structure function of these objects. This enables us to explore radio variability on timescales between several months and about five years. We find that, on these timescales, the mean structure function of the variable sources is consistent with a flat structure function. A plausible explanation to these observations is that a large fraction of the variability at $1.4 \mathrm{GHz}$ is induced by scintillations in the interstellar medium, rather than by intrinsic variability. Finally, for a subsample of the variables for which the redshift is available, we do not find strong evidence for a correlation between the variability amplitude and the source redshift.
\end{abstract}

Key words: ISM: general - quasars: general - radio continuum: general

Online-only material: color figures, machine-readable tables

\section{INTRODUCTION}

Variability of radio sources at low frequencies is mainly attributed to propagation effects (scintillations) induced by large-scale electron density inhomogeneities in the interstellar medium (ISM; e.g., Hunstead 1972; Rickett et al. 1984; Rickett 1990; Ghosh \& Rao 1992). The predicted variability structure function of compact radio sources due to scintillations (e.g., Blandford \& Narayan 1985; Goodman \& Narayan 1985; Blandford et al. 1986; Hjellming \& Narayan 1986) is roughly consistent with the typically observed structure function, at least below 5 GHz (e.g., Qian et al. 1995; Gaensler \& Hunstead 2000). These models predict a rise in the structure function up to timescales of $\sim 10$ days at $\approx 5 \mathrm{GHz}$ and up to $\sim 100$ days at $\approx 0.5 \mathrm{GHz}$, followed by a flattening of the structure function.

Specifically, Qian et al. (1995) analyzed radio observations of the compact radio source $1741-038(z=1.054)$ taken in several frequencies between 1.5 and $22 \mathrm{GHz}$. They compared the observed structure functions with theoretical models for scattering by an extended Galactic medium, with and without a thin screen component. They reported that for frequencies below about $5 \mathrm{GHz}$ the observations are consistent with a scattering by an extended Galactic medium and a thin screen. However, above this frequency they found excess variability relative to the models. Moreover, at these high frequencies, the structure function continues to rise towards longer timescales. They suggested that at high frequencies $(\gtrsim 5 \mathrm{GHz})$ some of the variability of this radio source is intrinsic. This general picture is also supported by Mitchell et al. (1994).

Gaensler \& Hunstead (2000) studied the variability of 55 radio calibrators observed by the Molonglo Observatory Synthesis Telescope (MOST) at $843 \mathrm{MHz}$. They constructed the structure function for 18 variable sources. For the majority of these variable objects the structure function flattens on timescales of a few hundreds days. Furthermore, they confirmed early results

\footnotetext{
3 Einstein fellow.
}

(Condon et al. 1979) which found that the variability amplitude is increasing as a function of the source spectral index $\alpha$, defined by $f_{v} \propto v^{\alpha}$, where $f_{v}$ is the specific flux at frequency $v$. This is attributed to a correlation between the source angular size and spectral index. Another confirmation for the importance of Galactic scintillations is that radio variability depends on Galactic latitude (e.g., Spangler et al. 1989; Ghosh \& Rao 1992; Gaensler \& Hunstead 2000). We note however that Rys \& Machalski (1990) did not find evidence for increasing fraction of $1.4 \mathrm{GHz}$ variability for sources brighter than $100 \mathrm{mJy}$ at low Galactic latitudes.

Lovell et al. (2008) presented results from the MicroArcsecond Scintillation-Induced Variability (MASIV) survey conducted at $5 \mathrm{GHz}$. Among their findings: half of the sources they monitored exhibit $2 \%-10 \%$ rms variations on timescales over two days. They also found that the structure function of the variable sources rises on timescales of a few days, and the variability amplitudes correlates with the $\mathrm{H} \alpha$ emission at the direction of the sources. Furthermore, there is evidence that the variability amplitude decreases with redshift above $z \approx 2$, presumably due to evolution of the source size with redshift (see, however, Lazio et al. 2008).

Here, we compare the two widest/deepest $1.4 \mathrm{GHz}$ sky surveys taken using the Very Large Array ${ }^{4}$ (VLA) and search for variable sources. We use these data sets to construct the average structure function on timescales between several months and about five years and to look for indications for intrinsic variability of these sources. In Section 2, we present augmented versions of the Faint Images of the Radio Sky at Twenty centimeters (FIRST) and NRAO VLA Sky Survey (NVSS) catalogs, which contain the mean time in which each source was observed. In Section 3 we cross correlate the two catalogs, and in Section 4 we construct the structure function of the variable sources. Finally, we discuss the results in Section 5.

\footnotetext{
4 The Very Large Array is operated by the National Radio Astronomy Observatory (NRAO), a facility of the National Science Foundation operated under cooperative agreement by Associated Universities, Inc.
} 
Table 1

Observing Times of NVSS Sources

\begin{tabular}{lcrcccc}
\hline \hline $\begin{array}{l}\text { J2000 R.A. } \\
(\mathrm{deg})\end{array}$ & $\begin{array}{c}\text { J2000 Decl. } \\
(\mathrm{deg})\end{array}$ & $\begin{array}{c}f \\
(\mathrm{mJy})\end{array}$ & $\begin{array}{c}\sigma_{\mathrm{f}} \\
(\mathrm{mJy})\end{array}$ & $N_{\text {obs }}$ & $\begin{array}{c}t_{\mathrm{ep}} \\
(\text { days })\end{array}$ & $\begin{array}{c}\delta t \\
(\text { days })\end{array}$ \\
\hline 194.89704 & -40.37908 & 3.60 & 0.70 & 1 & 0220.698 & 0.000 \\
249.00512 & -40.37366 & 99.70 & 3.60 & 1 & 0220.840 & 0.000 \\
199.42008 & -40.36441 & 24.30 & 1.20 & 1 & 0220.699 & 0.000 \\
248.90470 & -40.36169 & 3.00 & 0.60 & 1 & 0220.840 & 0.000 \\
212.86787 & -40.35236 & 2.70 & 0.50 & 1 & 0220.737 & 0.000 \\
\hline
\end{tabular}

Notes. A version of the NVSS catalog containing the source position, mean observing time $\left(t_{\mathrm{ep}}\right)$, number of scans $\left(N_{\mathrm{obs}}\right)$, and the time span over which the scans were obtained $(\delta t)$. The observing time, $t_{\mathrm{ep}}$, is given in JD -2450000 days, where JD is the Julian day. $f$ is the peak flux density and $\sigma_{\mathrm{f}}$ is the error in the peak flux density. We note that our cross correlation of the observing scans and the source catalog was not able to produce the observing times of 591 sources. The table is sorted by declination, therefore the sources listed here are near the edge of the survey footprint and have a single observation.

(This table is available in its entirety in a machine-readable form in the online journal. A portion is shown here for guidance regarding its form and content.)

Table 2

Observing Times of FIRST Sources

\begin{tabular}{lcccccc}
\hline \hline $\begin{array}{l}\text { J2000 R.A. } \\
(\mathrm{deg})\end{array}$ & $\begin{array}{c}\text { J2000 Decl. } \\
(\mathrm{deg})\end{array}$ & $\begin{array}{c}f \\
(\mathrm{mJy})\end{array}$ & $\begin{array}{c}\sigma_{\mathrm{f}} \\
(\mathrm{mJy})\end{array}$ & $N_{\text {obs }}$ & $\begin{array}{c}t_{\mathrm{ep}} \\
(\text { days })\end{array}$ & $\begin{array}{c}\delta t \\
(\text { days })\end{array}$ \\
\hline 354.74904 & -11.39882 & 1.68 & 0.14 & 1 & 0593.087 & 0.000 \\
5.19669 & -11.39761 & 1.02 & 0.15 & 1 & 0593.118 & 0.000 \\
6.82096 & -11.39611 & 1.11 & 0.15 & 1 & 0595.073 & 0.000 \\
359.90417 & -11.39585 & 1.39 & 0.15 & 1 & 0593.102 & 0.000 \\
6.70133 & -11.39458 & 1.17 & 0.14 & 1 & 0595.073 & 0.000 \\
\hline
\end{tabular}

Notes. As in Table 1 but for the FIRST catalog. We note that our cross correlation of the observing scans and the sources catalog was not able to produce the observing times of 379 sources.

(This table is available in its entirety in a machine-readable form in the online journal. A portion is shown here for guidance regarding its form and content.)

\section{THE CATALOGS}

The NVSS observations were carried out between 1993 June and 1999 April, while the FIRST survey observations were conducted between 1993 March and 2002 September. Therefore, these observations provide a long baseline to the structure function analysis. Constructing the structure function of variable sources requires knowledge of their fluxes at multiple epochs and the time at which the observations were taken. However, the NRAO VLA Sky Survey (NVSS; Condon et al. 1998) and the Faint Images of the Radio Sky at Twenty Centimeters (FIRST; Becker et al. 1995) source catalogs do not contain the time at which each source was observed. The reason for this is that the observing times are not well defined. Images in both surveys were taken by scanning the sky in a hexagonal grid in which observing points are separated by $26^{\prime}$. Both surveys were obtained using the VLA, in which the full width at half power at $1.4 \mathrm{GHz}$ is $\cong 31^{\prime}$. Each primary beam field of view was truncated to radii of $24^{\prime}$ and 23.5 for the NVSS and FIRST surveys, respectively. Therefore, each point on the sky effectively contains information from roughly four different pointings taken at different times.

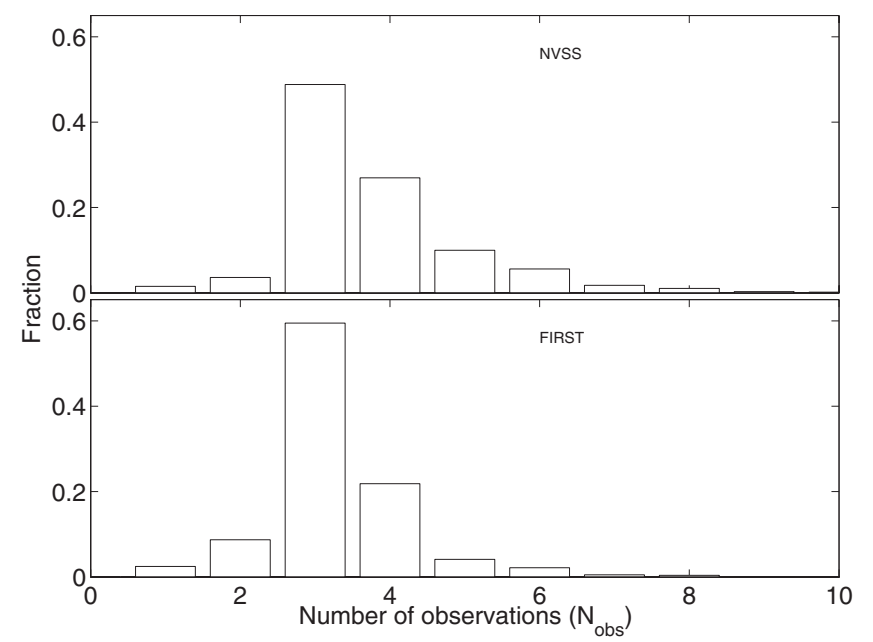

Figure 1. Histogram of the number of observations from which each source image was composed. The bin size is 1 observation. The upper panel is for NVSS sources, while the lower panel is for FIRST sources.

In order to obtain the epoch at which each source was observed we downloaded from the VLA archive ${ }^{5}$ the list of all observing scans which are associated with each sky survey. ${ }^{6}$

Next, we cross-correlate the list of observing scans for each project with its catalog of sources. We use a matching radii equal to the truncation radii of $24^{\prime}$ and 23.5 for the NVSS and FIRST ${ }^{7}$ surveys, respectively. This enable us to estimate for each source the number of observations $\left(N_{\mathrm{obs}}\right.$ ), its mean observing time (over all scan mid times, $t_{\mathrm{ep}}$ ) and the time span within which these observation were obtained $(\delta t)$.

The products are versions of the FIRST and NVSS catalogs that contain the observing time, number of observations, and time span of observations for each source. ${ }^{8}$ In Tables 1-2 we present a version of these catalogs containing the source coordinates and the observing time information for each source.

Figure 1 presents histograms of the number of individual snapshots used to compose each source image. For both surveys, the typical number of snapshots per composite image is 3-4 (see also Helfand et al. 1996). In Figure 2, we show histograms of $\delta t$ for the two surveys. This figure suggests that most of the images are made from snapshots taken within a few weeks of each other.

We note that a transient search based on the comparison of the FIRST and NVSS catalogs was presented in Levinson et al. (2002) and discussed in Gal-Yam et al. (2006) and Ofek et al. (2010). However, these previous efforts did not use the observing time of the sources.

\section{CROSS CORRELATION OF THE FIRST AND NVSS CATALOGS}

The FIRST catalog contains 816,331 sources, brighter than about $1 \mathrm{mJy}$, mainly in the North Galactic cap. About $81 \%$ of these sources have $\delta t<30$ days. The NVSS catalog contains $1,773,484$ objects with $\delta>-30 \mathrm{deg}$, brighter than about $3.5 \mathrm{mJy}$, of which $\cong 67 \%$ have $\delta t<30$ days. We select all the FIRST sources whose deconvolved major and minor axes

\footnotetext{
5 https://archive.nrao.edu/archive/advquery.jsp

6 These are observing code AC308 for the NVSS catalog and AB628, AB879 and $\mathrm{AB} 950$ for the FIRST catalog.

7 We use FIRST catalog version 20080716.

8 These are approximate observing times since we do not know if all the data was used in the reduction process of the FIRST and NVSS.
} 


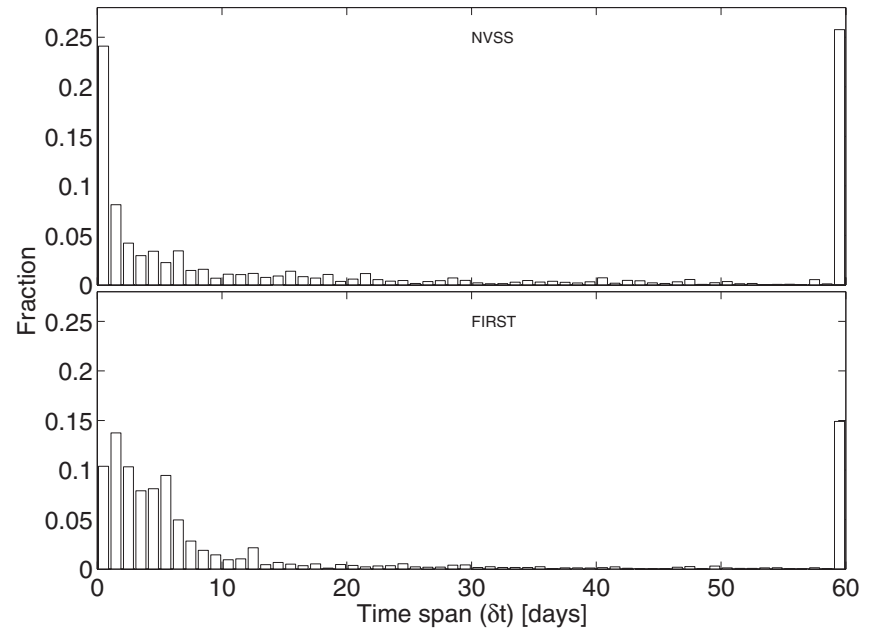

Figure 2. Histogram of the time span of observations from which each source image was composed $(\delta t)$. Bin size is 1 day. Panels as in Figure 1. The last bin on the right-hand side is for all $\delta t>59$ days.

equal zero (i.e., point sources), the peak flux density is larger than $5 \mathrm{mJy}$, all the scans composing their flux measurement $(\delta t)$ were taken within 30 days, and which are isolated from any other FIRST source (of any kind) within $60^{\prime \prime}$. Since the resolution of the NVSS is $45^{\prime \prime}$, nine times coarser than that of the FIRST survey, the last step is designed to remove NVSS sources whose flux may be contaminated by multiple FIRST objects. We found 6463 FIRST sources that satisfy these criteria. Next, from the NVSS catalog we select all sources with $\delta t<30$ days-we find $1,183,620$ sources that satisfy this criterion.

Then, for each object in the subset of the FIRST catalog we search for a source in the subset of the NVSS catalog which is found within $15^{\prime \prime}$ of the FIRST object. ${ }^{9}$ We found 4367 matched sources. These matches represent point sources for which we have both a FIRST and NVSS flux-density measurements. We note that only $68 \%$ of the FIRST sources in this list have NVSS matches. This is mostly due to the fact that we used only NVSS sources with $\delta t<30$ days.

Next, we would like to compare the fluxes of unresolved NVSS and FIRST sources. However, systematic biases in the NVSS and/or FIRST flux calibration could affect our analysis. Condon et al. (1998) and Becker et al. (1995) discussed photometric errors such as the CLEAN bias, and they made corrections to their source catalog flux densities. For both the NVSS and FIRST catalogs the CLEAN bias is $\approx-0.3 \mathrm{mJy}$, i.e., the same order as the rms noise in the images. There is also a well-known discrepancy between integrated fluxes of extended sources in FIRST and NVSS, owing to resolution effects (Blake $\&$ Wall 2002). In Figure 3 we show the mean of the peakflux ratio between matched individual NVSS and FIRST point sources, as a function of flux density (black circles).

This figure suggests that at the faint end, NVSS fluxes are systematically lower than FIRST fluxes. Blake \& Wall (2002) already reported this effect, although with an opposite direction and higher amplitude. However, Blake \& Wall (2002) looked at both resolved and unresolved sources, ${ }^{10}$ while we are interested only in point sources. Probably the most important reason for this trend is related to the fact that the NVSS and FIRST surveys have different resolutions. Another secondary effect is a bias

\footnotetext{
9 The median astrometric error for $5 \mathrm{mJy}$ sources in the NVSS catalog is about $3^{\prime \prime}$, and $99.4 \%$ of the errors of such sources are smaller than $15^{\prime \prime}$.

10 Dominated by resolved sources.
}

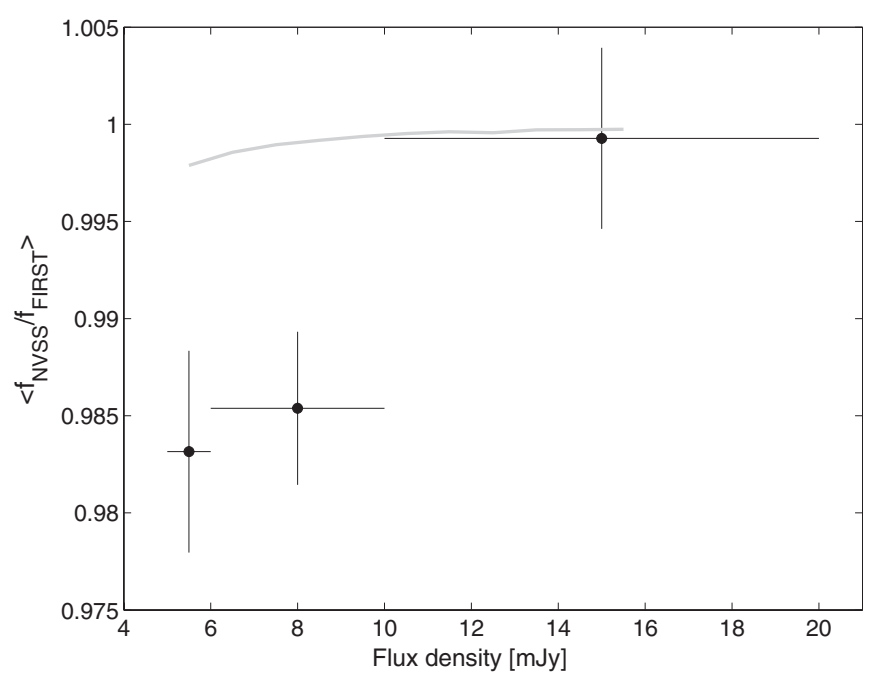

Figure 3. Mean of the flux ratio between matched individual NVSS and FIRST point sources, as a function of flux density (black circles). The horizontal "error bars" represent the bin size in which the mean flux ratio was measured. The expected amplitude of the Eddington-like bias (see the text) is shown by the gray line.

similar to the Eddington bias (Eddington 1913). This is because sources whose flux expectancy value is smaller than $5 \mathrm{mJy}$ (our FIRST flux cut) and are detected in the FIRST survey above $5 \mathrm{mJy}$ (due to measurement errors) have $>50 \%$ chance to have an NVSS flux density below $5 \mathrm{mJy}$. This effect is amplified by the fact that faint sources are more common, per unit flux density, than bright sources. The estimated amplitude of this bias, based on simulations, is shown by the gray line in Figure 3 .

Given these results we correct the NVSS fluxes of the matched point sources ${ }^{11}$ by the amount interpolated from the black circles in Figure 3. Above $20 \mathrm{mJy}$, we assume that the correction factor is 1 . We neglect the effect of the Eddington bias, since its expected amplitude is negligible (see Figure 3 ). We note that the maximum amplitude of the applied correction is smaller than the rms noise in the NVSS measurements and comparable to the rms noise of FIRST sources. A caveat in our bias analysis is that this bias may also depend on the actual angular extent of the unresolved sources. Therefore, the bias expectation value for a given flux may depend on a "hidden" parameter whose value is not measured, and cannot be entirely removed from the data.

The FIRST versus NVSS peak flux-density measurements of the matched point sources are presented in Figure 4. Based on this plot we estimate the standard deviation, $\sigma$, of the differences between the FIRST and NVSS specific fluxes as a function of FIRST flux. This is done by calculating the 68 percentile range in the flux-flux plot as a function of the FIRST flux density, $f_{\text {FIRST }}$. We divide the 68 percentile by 2 to estimate the standard deviation, and then fit a first order polynomial to the logarithm of the standard deviation estimator as a function of flux density. We find that the relative errors associated with these difference measurements are well represented by

$$
\sigma / f \approx 10^{-0.6652-0.0064 f_{\mathrm{FIRST}}} .
$$

Here we define variables as objects for which the FIRST versus NVSS flux difference is larger ${ }^{12}$ than $4 \sigma$. We found 43 such

\footnotetext{
11 The fluxes in Tables 1-2 are not corrected for this bias.

12 Assuming Gaussian noise, $4 \sigma$ corresponds to probability of $\cong 1 / 15,000$ while the number of measurements in our experiment (number of epochs multiplied by the number of sources) is 8734 .
} 


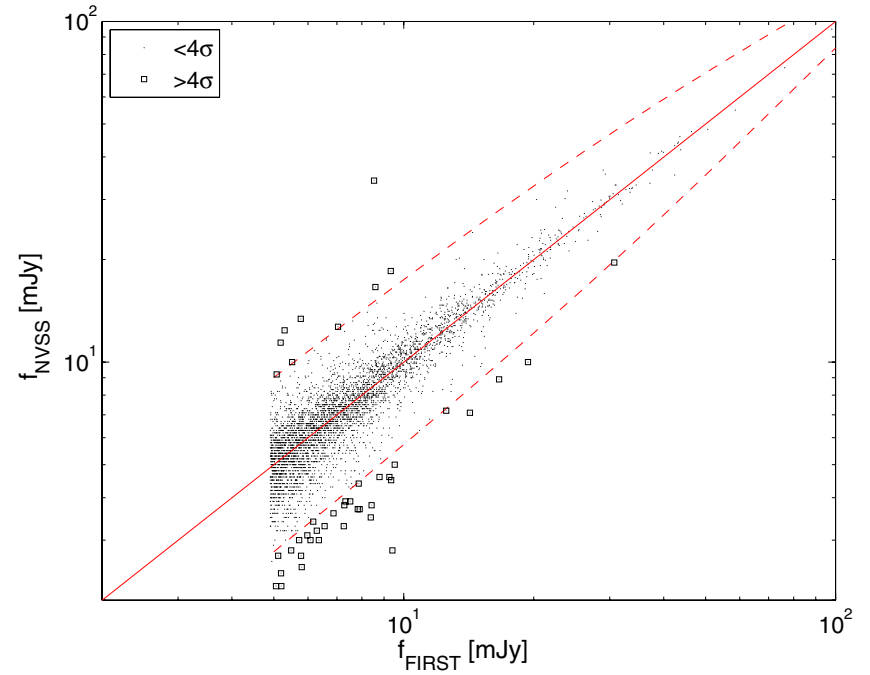

Figure 4. FIRST peak specific flux vs. the same quantity from the NVSS catalog for the 4367 matched unresolved sources (see the text). The solid line shows the 1:1 line, while the dashed lines represent $4 \sigma$ below and above the $1: 1$ line. Open boxes show the 43 sources that are variable by more than $4 \sigma$, while all the other matched sources are marked by dots. We note that there are more variable sources below the lower line than above the upper line (see the text).

(A color version of this figure is available in the online journal.)

variable sources, which are listed in Table 3 . We inspected the radio images of all these variable sources by eye, and comments on individual sources appear in this table. We note that the theoretical errors are smaller, and have different functional forms, than those implied by Equation (1). However, in order to avoid any possible uncertainties in the comparison between the two catalogs, we used the empirical errors.

Although we attempt to correct for the flux bias between FIRST and NVSS sources, in Figure 4 there are more variable sources below the lower $4 \sigma$ line than above the upper $4 \sigma$ line. This systematic difference may be related to the complexity of the bias between the FIRST and NVSS measurements, mentioned earlier. Effectively, this systematic bias induces errors in the number of "sigmas" in which a source is variable. However, the ratio of number of sources above the upper $4 \sigma$ line to that below the lower $4 \sigma$ line, is consistent with an additional systematic shift in the flux ratio of $\approx 0.35 \sigma$. Therefore, we conclude that since we used a relatively large variability threshold of $4 \sigma$, most of our variable sources are probably real.

\section{THE STRUCTURE FUNCTION}

Next, we calculate the mean structure function for all the 43 sources that exceed the $4 \sigma$ variability threshold in Figure 4. As a reference we also calculated the structure function for all the 3906 "non-variable" sources defined here as sources which variability is less than $2 \sigma$.

The structure function was calculated according to the following scheme. For each pair of matched FIRST and NVSS measurements, we calculate the time difference between the FIRST $\left(t_{\mathrm{ep}}^{\mathrm{FIRST}}\right)$ and NVSS $\left(t_{\mathrm{ep}}^{\mathrm{NVSS}}\right)$ epochs: $\Delta t_{i}=t_{\mathrm{ep}}^{\mathrm{FIRST}}-t_{\mathrm{ep}}^{\mathrm{NVSS}}$. Here, $i$ is the index of the pair. We also calculate for each pair:

$$
\Delta f_{i} / \bar{f}_{i}=\frac{f_{\mathrm{NVSS}, i}-\bar{f}_{i}}{\bar{f}_{i}},
$$

where $f_{\mathrm{NVSS}, i}$ and $f_{\mathrm{FIRST}, i}$ are the NVSS and FIRST specific fluxes of the $i$-th source, and $\bar{f}_{i}=\left(f_{\mathrm{NVSS}, i}+f_{\mathrm{FIRST}, i}\right) / 2$. Next,

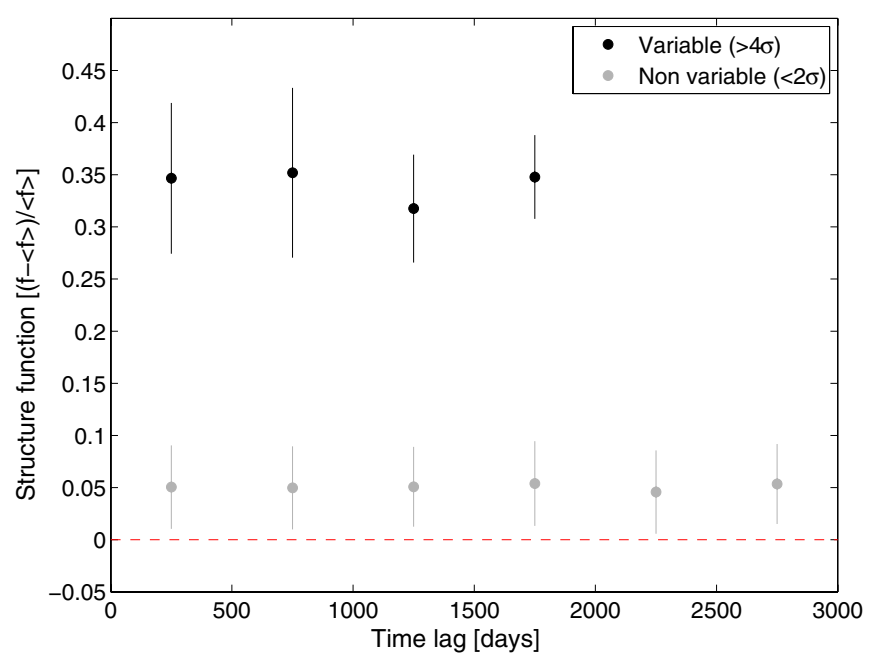

Figure 5. Structure function of variable sources (black circles) and non-variable sources (gray circles) as a function of time lag. The measurements are displayed only for bins in which the number of flux differences is larger than 4 . The errors are based on the standard deviation in each bin. The horizontal dashed line marks the zero structure function.

(A color version of this figure is available in the online journal.)

the structure function and its error are estimated in bins of 500 days, between zero and 3500 days, by calculating the mean and standard deviation of $\Delta f_{i} / \bar{f}_{i}$ for all the pairs $i$ in the appropriate bin.

Figure 5 shows the structure function for the variable (black) and non variable (gray) sources. The structure function in this time range is consistent with being flat, with mean relative variability of about $35 \%$. However, the value of $35 \%$ probably represents our sensitivity for variability rather than some physical variability level. The only physically interesting fact is the flatness of the structure function.

\section{DISCUSSION}

We present versions of the FIRST and NVSS catalogs that contain the mean epoch in which each source was observed. We use these catalogs to look for variable sources, and we construct the structure function for these objects. We show that the structure function is flat on timescales between about half a year and five years.

It is well known that the structure function of variable radio sources rises on timescales of days to tens of days (e.g., Qian et al. 1995; Gaensler \& Hunstead 2000; Lovell et al. 2008; Ofek et al. 2011). The intrinsic variability of compact radio sources, which are mainly active galactic nuclei (AGNs), on days timescales would imply that the sources have small physical size. This in turn requires a very high rest-frame brightness temperature $\left(T_{\mathrm{B}, \text { rest }}\right)$ orders of magnitude above $\sim 10^{12} \mathrm{~K}$, which is the limit for an incoherent synchrotron source (e.g., Kellermann \& Pauliny-Toth 1969; Readhead 1994). Therefore, most or all of the variability of radio sources, below $\sim 5 \mathrm{GHz}$, on these short timescales is presumably due to scintillations in the ISM. Moreover, based on causality arguments, intrinsic variability of compact radio sources is expected only on timescales $\left(\tau_{V}\right)$ larger than

$$
\begin{aligned}
\tau_{V} \gtrsim & 50\left(\frac{\Delta f}{1 \mathrm{mJy}}\right)^{1 / 2}\left(\frac{d_{\text {lum }}}{6.7 \mathrm{Gpc}}\right)\left(\frac{v}{1.4 \mathrm{GHz}}\right)^{-1} \\
& \times\left(\frac{\mathcal{D}}{10}\right)^{-3 / 2}\left(\frac{1+z}{2}\right)^{3 / 2}\left(\frac{T_{\mathrm{B}, \text { rest }}}{10^{12} \mathrm{~K}}\right)^{-1 / 2} \text { days }
\end{aligned}
$$


Table 3

Variable Sources

\begin{tabular}{|c|c|c|c|c|c|c|c|c|c|c|c|c|c|c|}
\hline \multicolumn{2}{|c|}{$\mathrm{J} 2000.0$} & \multirow[b]{2}{*}{$\begin{array}{l}f_{\text {FIRST }} \\
(\mathrm{mJy})\end{array}$} & \multirow[b]{2}{*}{$\begin{array}{l}f_{\mathrm{NVSS}} \\
(\mathrm{mJy})\end{array}$} & \multirow[b]{2}{*}{$\begin{array}{c}\Delta t \\
\text { (day) }\end{array}$} & \multicolumn{2}{|c|}{ USNO-B1 } & \multicolumn{3}{|c|}{ 2MASS } & \multirow{2}{*}{$\begin{array}{c}\text { ROSAT } \\
\text { Dist } \\
\left({ }^{\prime \prime}\right)\end{array}$} & \multicolumn{4}{|c|}{ SDSS } \\
\hline $\begin{array}{l}\text { R.A. } \\
\text { (deg) }\end{array}$ & $\begin{array}{l}\text { Decl. } \\
\text { (deg) }\end{array}$ & & & & $\begin{array}{c}B_{2} \\
(\mathrm{mag})\end{array}$ & $\begin{array}{c}R_{2} \\
(\mathrm{mag})\end{array}$ & $\begin{array}{c}J \\
(\mathrm{mag})\end{array}$ & $\begin{array}{c}H \\
\text { (mag) }\end{array}$ & $\begin{array}{c}K \\
(\mathrm{mag})\end{array}$ & & $\begin{array}{c}g \\
(\mathrm{mag})\end{array}$ & $\begin{array}{c}r \\
(\mathrm{mag})\end{array}$ & $z_{\mathrm{sp}}$ & $z_{\mathrm{ph}}$ \\
\hline 229.95525 & -5.90828 & $19.40 \pm 0.14$ & $10.0 \pm 0.5$ & 1736.7 & $\ldots$ & $\ldots$ & $\ldots$ & $\ldots$ & $\ldots$ & $\ldots$ & $\ldots$ & $\cdots$ & $\ldots$ & \\
\hline 34.81875 & -4.64270 & $5.72 \pm 0.14$ & $3.0 \pm 0.7$ & 1270.9 & $\ldots$ & $\ldots$ & $\ldots$ & $\ldots$ & $\ldots$ & $\ldots$ & $\ldots$ & $\ldots$ & $\ldots$ & $\ldots$ \\
\hline 208.61233 & 0.73462 & $5.20 \pm 0.18$ & $2.4 \pm 0.5$ & 760.9 & $\ldots$ & $\ldots$ & $\ldots$ & $\ldots$ & $\ldots$ & $\ldots$ & $\ldots$ & $\ldots$ & $\ldots$ & $\ldots$ \\
\hline 120.64970 & 7.55780 & $5.19 \pm 0.15$ & $11.4 \pm 1.5$ & 1758.9 & $\ldots$ & $\ldots$ & $\ldots$ & $\ldots$ & $\ldots$ & $\ldots$ & . & $\ldots$ & $\ldots$ & $\ldots$ \\
\hline 193.96310 & 8.74396 & $7.90 \pm 0.19$ & $3.7 \pm 0.4$ & 1288.4 & $\ldots$ & $\ldots$ & $\ldots$ & $\ldots$ & $\ldots$ & $\ldots$ & 24.70 & 22.76 & $\ldots$ & 0.30 \\
\hline 158.12037 & 8.98541 & $60 \pm 0.15$ & $16.6 \pm 0.6$ & 1719.3 & 19.6 & 18.5 & $\ldots$ & $\ldots$ & $\ldots$ & $\ldots$ & 19.28 & 19.28 & 0.454 & 0.45 \\
\hline 155.94935 & 10.65521 & $.77 \pm 0.15$ & $13.4 \pm 1.6$ & 1698.6 & $\ldots$ & $\ldots$ & $\ldots$ & $\ldots$ & $\ldots$ & $\ldots$ & 24.42 & 22.33 & $\ldots$ & $\ldots$ \\
\hline 225.14947 & 13.45289 & $08 \pm 0.14$ & $3.0 \pm 0.4$ & 1271.5 & $\ldots$ & 20.0 & $\ldots$ & $\ldots$ & $\ldots$ & $\ldots$ & 23.01 & 21.37 & $\ldots$ & \\
\hline 114.80608 & 18.03965 & $26 \pm 0.16$ & $3.3 \pm 0.5$ & 1762.5 & 17.1 & 16.9 & 16.14 & 15.49 & 14.86 & $\ldots$ & 17.36 & 17.12 & $\ldots$ & 1.00 \\
\hline 201.32666 & 19.46406 & $.04 \pm 0.14$ & $12.7 \pm 0.5$ & 1712.8 & $\ldots$ & $\ldots$ & $\ldots$ & $\ldots$ & $\ldots$ & $\ldots$ & $\ldots$ & $\ldots$ & $\ldots$ & $\ldots$ \\
\hline 206.50009 & 19.73055 & $78 \pm 0.15$ & $2.7 \pm 0.4$ & 1702.0 & $\ldots$ & $\ldots$ & $\ldots$ & $\ldots$ & $\ldots$ & $\ldots$ & 24.12 & 23.18 & $\ldots$ & $\ldots$ \\
\hline 244.32375 & 20.57935 & $82 \pm 0.14$ & $.7 \pm 0.4$ & 1320.1 & $\ldots$ & $\ldots$ & $\ldots$ & $\ldots$ & $\ldots$ & $\ldots$ & 22.70 & 22.29 & $\ldots$ & $\ldots$ \\
\hline 222.17540 & 20.67543 & $88 \pm 0.15$ & $3.6 \pm 0.5$ & 1322.1 & $\ldots$ & $\ldots$ & $\ldots$ & $\ldots$ & $\ldots$ & $\ldots$ & $\ldots$ & $\ldots$ & $\ldots$ & $\ldots$ \\
\hline 247.57423 & 23.33178 & $41 \pm 0.36$ & $2.8 \pm 0.4$ & 286.9 & 20.9 & 19.7 & $\ldots$ & $\ldots$ & $\ldots$ & $\ldots$ & 20.87 & 20.52 & $\ldots$ & 2.60 \\
\hline 212.99535 & 23.89795 & $9.53 \pm 0.14$ & $5.0 \pm 0.4$ & 284.4 & $\ldots$ & $\ldots$ & $\ldots$ & $\ldots$ & $\ldots$ & $\ldots$ & 22.19 & 21.96 & $\ldots$ & $\ldots$ \\
\hline 252.75117 & 24.26111 & $8.79 \pm 0.14$ & $4.6 \pm 0.4$ & 197.4 & $\ldots$ & $\ldots$ & $\ldots$ & $\ldots$ & $\ldots$ & $\ldots$ & 22.86 & 21.67 & $\ldots$ & $\ldots$ \\
\hline 190.23317 & 24.33961 & $80 \pm 0.15$ & $2.5 \pm 0.4$ & 284.2 & $\ldots$ & $\ldots$ & $\ldots$ & $\ldots$ & $\ldots$ & $\ldots$ & $\ldots$ & $\ldots$ & $\ldots$ & $\ldots$ \\
\hline 182.98029 & 25.36394 & $48 \pm 0.15$ & $2.8 \pm 0.4$ & 213.8 & 20.2 & 20.5 & $\ldots$ & $\ldots$ & $\ldots$ & $\ldots$ & 20.83 & 20.66 & $\ldots$ & 0.30 \\
\hline 247.48198 & 27.72523 & $28 \pm 0.16$ & $.8 \pm 0.5$ & 183.4 & $\ldots$ & 19.7 & & $\ldots$ & $\ldots$ & $\ldots$ & 22.40 & 20.78 & $\ldots$ & $\ldots$ \\
\hline 238.73764 & 29.95945 & $5.08 \pm 0.14$ & $9.2 \pm 1.0$ & -754.7 & 18.9 & 18.7 & $\ldots$ & $\ldots$ & $\ldots$ & $\ldots$ & 18.89 & 18.87 & 0.855 & 0.85 \\
\hline 203.08994 & 29.99894 & $16.62 \pm 0.59$ & $8.9 \pm 1.1$ & -745.7 & $\ldots$ & $\ldots$ & $\ldots$ & $\ldots$ & $\ldots$ & & $\ldots$ & $\ldots$ & $\ldots$ & $\ldots$ \\
\hline 136.90323 & 30.2587 & & .5 & -28 & $\ldots$ & $\ldots$ & $\ldots$ & $\ldots$ & $\ldots$ & $\ldots$ & 22.13 & 21.99 & $\ldots$ & $\ldots$ \\
\hline 203.03348 & 30.6911 & 1.35 & $6 \pm 0.5$ & -735 & $\ldots$ & $\ldots$ & $\ldots$ & $\ldots$ & $\ldots$ & $\ldots$ & $\ldots$ & $\ldots$ & $\ldots$ & $\ldots$ \\
\hline 169.75685 & 36.87706 & .15 & 18.5 & -289.7 & 16.3 & 15.7 & 15.98 & 15.25 & 14.60 & $\ldots$ & $\ldots$ & 19.25 & $\ldots$ & $\ldots$ \\
\hline 170.99205 & 38.43646 & $6.17 \pm 0.15$ & $3.4 \pm 0.4$ & -260.7 & $\ldots$ & $\ldots$ & $\ldots$ & $\ldots$ & $\ldots$ & $\ldots$ & $\ldots$ & $\ldots$ & $\ldots$ & $\ldots$ \\
\hline 122.43647 & 38.50171 & $7.86 \pm 0.14$ & $4.4 \pm 0.5$ & 202.8 & $\ldots$ & $\ldots$ & $\ldots$ & $\ldots$ & $\ldots$ & $\ldots$ & $\ldots$ & $\ldots$ & $\ldots$ & $\ldots$ \\
\hline 119.02080 & 39.25484 & $6.36 \pm 0.14$ & $3.0 \pm 0.4$ & 218.6 & 19.2 & 17.6 & $\ldots$ & $\ldots$ & $\ldots$ & $\ldots$ & 20.45 & 19.62 & $\ldots$ & $\ldots$ \\
\hline 262.32334 & 44.77948 & $5.98 \pm 0.14$ & $3.1 \pm 0.5$ & 700.7 & $\ldots$ & $\ldots$ & $\ldots$ & $\ldots$ & $\ldots$ & $\ldots$ & $\ldots$ & $\ldots$ & $\ldots$ & $\ldots$ \\
\hline 229.90208 & 44.97024 & $5.12 \pm 0.15$ & $2.7 \pm 0.4$ & 710.8 & $\ldots$ & $\ldots$ & $\ldots$ & $\ldots$ & $\ldots$ & $\ldots$ & 24.15 & 23.91 & $\ldots$ & 0.10 \\
\hline 134.99454 & 45.87719 & $30.74 \pm 0.15$ & $19.6 \pm 0.7$ & 1207.4 & 19.2 & 18.6 & $\ldots$ & $\ldots$ & $\ldots$ & $\ldots$ & 18.86 & 19.05 & 0.440 & 0.50 \\
\hline 220.45533 & 50.71170 & $5.06 \pm 0.16$ & $2.2 \pm 0.4$ & & $\ldots$ & $\ldots$ & $\ldots$ & $\ldots$ & $\ldots$ & $\ldots$ & $\ldots$ & $\ldots$ & $\ldots$ & $\ldots$ \\
\hline 262.65758 & 52.24396 & $32 \pm 0.14$ & $9 \pm 0.4$ & 766 & & & 16.57 & 15.86 & 15.01 & $\ldots$ & $\cdots$ & $\ldots$ & $\ldots$ & $\ldots$ \\
\hline 211.65318 & 52.6847 & & & & $\ldots$ & $\ldots$ & $\ldots$ & $\ldots$ & $\ldots$ & $\ldots$ & $\ldots$ & $\ldots$ & $\ldots$ & $\ldots$ \\
\hline 159.71340 & 53.49093 & $.53 \pm 0.15$ & $34.1 \pm 1.8$ & 765.5 & 19.9 & $\ldots$ & $\ldots$ & $\ldots$ & $\ldots$ & 53.2 & $\ldots$ & $\ldots$ & $\ldots$ & $\ldots$ \\
\hline 226.56440 & 53.92288 & $5.30 \pm 0.15$ & $12.4 \pm 1.1$ & 770.9 & 21.6 & $\ldots$ & $\ldots$ & $\ldots$ & $\ldots$ & $\ldots$ & 21.75 & 21.57 & $\ldots$ & 2.40 \\
\hline 179.14175 & 54.63838 & $12.54 \pm 0.27$ & $7.2 \pm 0.5$ & 779.5 & $\ldots$ & $\ldots$ & $\ldots$ & $\ldots$ & $\ldots$ & $\ldots$ & $\ldots$ & $\ldots$ & $\ldots$ & $\ldots$ \\
\hline 157.78099 & 56.75153 & $6.56 \pm 0.18$ & $3.3 \pm 0.4$ & 780.2 & 20.6 & 18.9 & $\ldots$ & $\ldots$ & $\ldots$ & $\ldots$ & 20.84 & 19.82 & $\ldots$ & $\ldots$ \\
\hline 157.96070 & 56.85260 & $6.29 \pm 0.20$ & $3.2 \pm 0.4$ & 780.2 & $\ldots$ & $\ldots$ & $\ldots$ & $\ldots$ & $\ldots$ & $\ldots$ & $\ldots$ & $\ldots$ & $\ldots$ & $\ldots$ \\
\hline 160.55682 & 59.35609 & $5.51 \pm 0.15$ & $10.0 \pm 0.9$ & 2642.4 & $\ldots$ & $\ldots$ & $\ldots$ & $\ldots$ & $\ldots$ & $\ldots$ & $\ldots$ & $\ldots$ & $\ldots$ & $\ldots$ \\
\hline 151.08074 & 59.53517 & $8.38 \pm 0.16$ & $3.5 \pm 0.4$ & 3075.2 & $\ldots$ & $\ldots$ & $\ldots$ & $\ldots$ & $\ldots$ & $\ldots$ & $\ldots$ & $\ldots$ & $\ldots$ & $\ldots$ \\
\hline 185.45517 & 61.59247 & $7.51 \pm 0.15$ & $3.9 \pm 0.4$ & 2655.3 & $\ldots$ & & $\ldots$ & $\ldots$ & $\ldots$ & $\ldots$ & 23.10 & 21.16 & $\ldots$ & $\ldots$ \\
\hline 248.86649 & 62.64994 & $14.23 \pm 1.51$ & $7.1 \pm 1.1$ & 2665.3 & & & $\ldots$ & $\ldots$ & $\ldots$ & $\ldots$ & $\ldots$ & $\ldots$ & $\ldots$ & $\ldots$ \\
\hline 124.64543 & 63.90501 & $5.21 \pm 0.14$ & $2.2 \pm 0.4$ & 3192.3 & $\ldots$ & $\ldots$ & $\ldots$ & $\ldots$ & $\ldots$ & $\ldots$ & $\ldots$ & $\ldots$ & $\ldots$ & $\ldots$ \\
\hline
\end{tabular}

Notes. List of 43 sources which vary by more than $4 \sigma$ between the FIRST and NVSS epochs. The table is sorted by declination. Column descriptions: $f$ is the peak specific flux and its error. The subscript indicate the catalog name. $\Delta t$ is the time between the FIRST and NVSS observations. The position of each source was cross correlated with various catalogs, including the USNO-B1 (Monet et al. 2003), 2MASS (Skrutskie et al. 2006), ROSAT bright and faint source catalogs (Voges et al. 1999, 2000), and the Sloan Digital Sky Survey (York et al. 2000). In case counterparts are found we list their USNO-B1 $B_{2}$ and $R_{2}$ magnitudes, 2MASS $J, H$, and $K$ magnitudes, distance from ROSAT source, and SDSS $g$ and $r$ point-spread function magnitudes and redshifts. We use search radius, relative to the FIRST catalog position, of $60^{\prime \prime}$ for ROSAT and 2". 5 for all the other catalogs. $z$ is the SDSS spectroscopic redshift of the source, while $z_{\mathrm{ph}}$ is the photometric redshift of the source based on the SDSS colors. We use a photometric redshift estimator for quasars which is described in Ofek et al. (2002). The photometric redshift is calculated only if the source is indicated as a possible quasar in the SDSS database. Sources number 2, 4, 20, 24, and 39 are indicated as complex sources with a high peak in the NVSS catalog. We note that these sources are brighter in the NVSS catalog than in the FIRST catalog. Comments on individual sources:

R.A. $=155.94935 \mathrm{deg}$, Decl. $=+10.65521 \mathrm{deg}$ : The peak flux we measure in the NVSS image is a factor of two lower than the flux stated in the NVSS catalog, so this may be a constant source.

R.A. $=247.57423 \mathrm{deg}$, Decl. $=+23.33178 \mathrm{deg}$ : Near the strong source 3C 340—NVSS and FIRST images are noisy.

R.A. $=203.03348$ deg, Decl. $=+30.69115$ deg: Radio images are noisy.

R.A. $=159.71340 \mathrm{deg}$, Decl. $=+53.49093 \mathrm{deg}$ : This is possibly a radio supernova in the outskirts of NGC 3310 (Argo et al. 2004).

R.A. $=226.56440 \mathrm{deg}$, Decl. $=+53.92288 \mathrm{deg}$ : NVSS image shows a double source.

R.A. $=157.78099 \mathrm{deg}$, Decl. $=+56.75153 \mathrm{deg}$ : Extended emission 15 arcmin from source.

R.A. $=157.96070 \mathrm{deg}$, Decl. $=+56.85260 \mathrm{deg}$ : Extended emission 4 arcmin from source.

R.A. $=124.64543 \mathrm{deg}$, Decl. $=+63.90501 \mathrm{deg}$ : FIRST flux may be influenced by sidelobes from 3C 343 ( $4.5 \mathrm{Jy}, 9$ arcmin to the NW). 


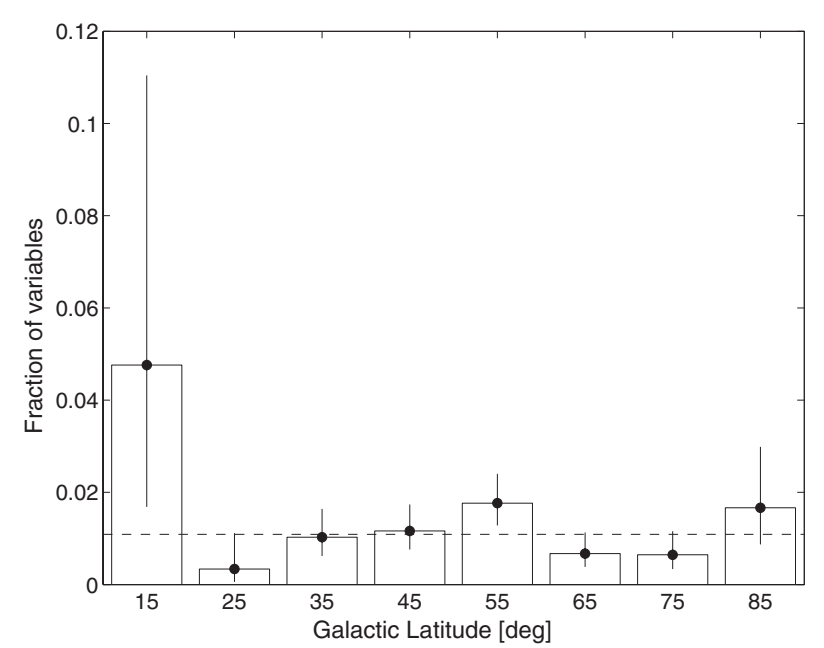

Figure 6. Fraction of variables, relative to all the non-variable and variable sources, as a function of Galactic latitude. The error bars represent the $1 \sigma$ errors on the fractions (Gehrels 1986). The dashed horizontal line marks the mean "all-sky" fraction of variables in our sample (i.e., 43/3949 = 0.0109).

where $\Delta f$ is the variation amplitude in specific flux, $d_{\text {lum }}$ is the luminosity distance (normalized at $z \cong 1$ ), $\mathcal{D}$ is the Doppler factor of a relativistic motion in the source, and $z$ is the source redshift. In Equation (3), $T_{\mathrm{B} \text {, rest }}$ is given in the rest frame and all the other parameters are in the observer reference frame. We note that Equation (3) is derived from the relation for the brightness temperature, and replacing the source size by $c \tau_{V}$, where $c$ is the speed of light.

In contrast, observations of quasars and BL Lac objects performed in the $4.8-14.5 \mathrm{GHz}$ range showed that the structure function saturates only on timescales between a year and ten years (Hughes et al. 1992). Therefore, the fact that we do not see any significant rise in the structure function on timescales of months to years suggests that, at $1.4 \mathrm{GHz}$, the amplitude of intrinsic variability relative to scintillations is small. Alternatively, it may suggest that the $1.4 \mathrm{GHz}$ power spectrum of AGN radio variability is consistent with a white-noise power spectrum, rather than the red-noise power spectrum typically seen at shorter wavelengths (e.g., Giveon et al. 1999; Markowitz et al. 2003). We note that Padrielli et al. (1987) reported on a class of sources (denoted "C-BBV" in their terminology) which show correlated intrinsic variability in low $(0.4 \mathrm{GHz})$ and high $(14.5 \mathrm{GHz})$ frequencies. However, these sources are a minority among the variable sources in their sample.

Figure 6 shows the fraction of variables as a function of Galactic latitude. The first bin contains two variables out of 42 objects (a fraction of 0.048). However, the expectation value in this bin, estimated based on the mean fraction of variables (dashed line in Figure 6; 0.0109) multiplied by the number of sources in the first bin (42) is 0.457. Assuming a binomial distribution, the cumulative probability to observe $\geqslant 2$ events, given an expectation value of 0.457 , is $7.7 \%$. This rules out the null hypothesis that the low-latitude variable-fraction is drawn from a uniform all-sky distribution at the $92.3 \%$ confidence. Therefore, a larger sample is required in order to confirm the earlier claims that the fraction of variables is larger at low Galactic latitude (e.g., Gaensler \& Hunstead 2000). If this excess is real, then a plausible explanation is that it is due to ISM scintillations which are more prominent at low Galactic latitudes. However, we cannot rule out that some of this excess in variability is due to a population of Galactic variable sources as suggested by Becker et al. (2010).

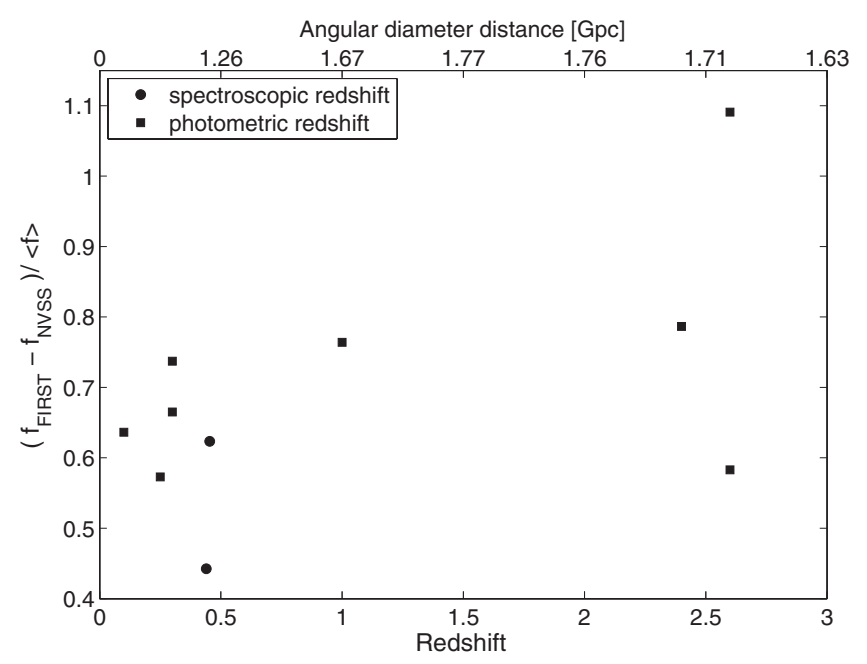

Figure 7. Variability relative amplitude, $\Delta f_{i} / \bar{f}_{i}$, as a function of redshift for all nine variable sources that are quasars or quasar candidates (Table 3 ). The upper $x$-axis shows the angular diameter distance corresponding to the redshift, assuming WMAP fifth-year cosmological parameters (Komatsu et al. 2009).

Finally, we use our dataset to look for correlation of the variability amplitude with redshift. There are several factors that can contribute to such a correlation. For example, scintillations depends on the source angular size (larger amplitude for smaller sources), intrinsic source size evolution, broadening due to scattering, and maybe even scintillation in the intra-galactic medium (which may depend on the He re-ionization). Figure 7 shows $\Delta f_{i} / \bar{f}_{i}$ as a function of redshift for all nine variable sources for which we have a redshift estimate (Table 3 ). The Spearman rank (Pearson) correlation coefficient between the redshift and the relative variability amplitude is $0.59(0.79)$. In order to estimate the significance of this correlation we conduct $10^{4}$ bootstrap simulations (Efron 1982; Efron \& Tibshirani 1993). In each simulation, we select, for each source redshift, a random variability amplitude from the list of nine amplitudes. We find that the probability to get a Spearman rank correlation coefficient $>0.59$ is about $5 \%$. Therefore, unlike Lovell et al. (2008) we do not find any strong evidence for correlation between scintillations and redshift. However, our sample is considerably smaller than the one presented by Lovell et al. (2008). We note that eight out of the nine sources in Figure 7 are found above Galactic latitude of $40 \mathrm{deg}$, and one source is at Galactic latitude of about $18 \mathrm{deg}$. Removing the single low galactic latitude source (the source at $z \approx 1$ in Figure 7 ) reduces the Spearman rank correlation to 0.42 and therefore does not change this results significantly.

We thank Orly Gnat for reading the manuscript and to Jim Condon, Heinz Andernach, and an anonymous referee for their comments. E.O.O. is supported by an Einstein fellowship and NASA grants. This paper is based on observations conducted with the VLA, which is operated by the National Radio Astronomy Observatory (NRAO), a facility of the National Science Foundation operated under cooperative agreement by Associated Universities, Inc.

\section{REFERENCES}

Argo, M. K., Muxlow, T. W. B., Pedlar, A., Beswick, R. J., \& Strong, M. 2004, MNRAS, 351, L66

Becker, R. H., Helfand, D. J., White, R. L., \& Proctor, D. D. 2010, AJ, 140, 157

Becker, R. H., White, R. L., \& Helfand, D. J. 1995, ApJ, 450, 559

Blake, C., \& Wall, J. 2002, MNRAS, 337, 993 
Blandford, R., \& Narayan, R. 1985, MNRAS, 213, 591

Blandford, R., Narayan, R., \& Romani, R. W. 1986, ApJ, 301, L53

Condon, J. J., Cotton, W. D., Greisen, E. W., Yin, Q. F., Perley, R. A., Taylor, G. B., \& Broderick, J. J. 1998, AJ, 115, 1693

Condon, J. J., Ledden, J. E., Odell, S. L., \& Dennison, B. 1979, AJ, 84, 1

Eddington, A. S. 1913, MNRAS, 73, 359

Efron, B. 1982, The Jackknife, the Bootstrap and Other Resampling Plans, CBMS-NSF Regional Conf. Ser. in Applied Mathematics (Philadelphia, PA: SIAM)

Efron, B., \& Tibshirani, R. J. 1993, An Introduction to the Bootstrap, Monographs on Statistics and Applied Probability 57 (London: Chapman \& Hall/ CRC)

Gaensler, B. M., \& Hunstead, R. W. 2000, PASA, 17, 72

Gal-Yam, A., et al. 2006, ApJ, 639, 331

Gehrels, N. 1986, ApJ, 303, 336

Ghosh, T., \& Rao, A. P. 1992, A\&A, 264, 203

Giveon, U., Maoz, D., Kaspi, S., Netzer, H., \& Smith, P. S. 1999, MNRAS, 306, 637

Goodman, J., \& Narayan, R. 1985, MNRAS, 214, 519

Gregory, P. C., Scott, W. K., Douglas, K., \& Condon, J. J. 1996, ApJS, 103, 427

Helfand, D. J., Das, S. R., Becker, R. H., White, R. L., \& McMahon, R. G. 1996, in ASP Conf. Ser. 110, Blazar Continuum Variability, ed. H. R. Miller, J. R. Webb, \& J. C. Noble (San Francisco, CA: ASP), 214

Hjellming, R. M., \& Narayan, R. 1986, ApJ, 310, 768

Hughes, P. A., Aller, H. D., \& Aller, M. F. 1992, ApJ, 396, 469

Hunstead, R. W. 1972, Astrophys. Lett., 12, 193
Kellermann, K. I., \& Pauliny-Toth, I. I. K. 1969, ApJ, 155, L71

Komatsu, E., et al. 2009, ApJS, 180, 330

Lazio, T. J. W., Ojha, R., Fey, A. L., Kedziora-Chudczer, L., Cordes, J. M. Jauncey, D. L., \& Lovell, J. E. J. 2008, ApJ, 672, 115

Levinson, A., Ofek, E. O., Waxman, E., \& Gal-Yam, A. 2002, ApJ, 576, 923

Lovell, J. E. J., et al. 2008, ApJ, 689, 108

Markowitz, A., et al. 2003, ApJ, 593, 96

Mitchell, K. J., Dennison, B., Condon, J. J., Altschuler, D. R., Payne, H. E., O’dell, S. L., \& Broderick, J. J. 1994, ApJS, 93, 441

Monet, D. G., et al. 2003, AJ, 125, 984

Ofek, E. O., Breslauer, B., Gal-Yam, A., Frail, D., Kasliwal, M. M., Kulkarni, S. R., \& Waxman, E. 2010, ApJ, 711, 517

Ofek, E. O., Rix, H.-W., Maoz, D., \& Prada, F. 2002, MNRAS, 337, 1163

Ofek, E. O., et al. 2011, ApJ, submitted (arXiv:1103.3010)

Padrielli, L., et al. 1987, A\&AS, 67, 63

Qian, S. J., Britzen, S., Witzel, A., Krichbaum, T. P., Wegner, R., \& Waltman, E. 1995, A\&A, 295, 47

Readhead, A. C. S. 1994, ApJ, 426, 51

Rickett, B. J. 1990, ARA\&A, 28, 561

Rickett, B. J., Coles, W. A., \& Bourgois, G. 1984, A\&A, 134, 390

Rys, S., \& Machalski, J. 1990, A\&A, 236, 15

Skrutskie, M. F., et al. 2006, AJ, 131, 1163

Spangler, S., Fanti, R., Gregorini, L., \& Padrielli, L. 1989, A\&A, 209, 315

Voges, W., et al. 1999, A\&A, 349, 389

Voges, W., et al. 2000, IAU Circ., 7432, 3

York, D. G., et al. 2000, AJ, 120, 1579 